\title{
Preface
}

No one knows at the present time if higher spin gauge fields have anything to do with reality. But the subject is interesting in itself, and - as its founder P. A. M. Dirac wrote in 1936 - it might be good to have a theory at hand if they do turn out relevant for fundamental physics [1]. ${ }^{1}$ Dirac studied massive fields, and it took another 40 years for massless higher spin gauge fields to come into focus with the work of C. Fronsdal in 1978 [3]. The subject is now in its ninth decade. This is a first book in a planned two volume project aimed at trying to cover some parts of this fascinating subject.

\section{Origin of the subject}

The theory of higher spin fields dates back to the beginnings of quantum field theory in the 1930s. For a long time, the focus was on massive fields, as such could describe massive matter particles with spin. From Wigner's classification of the representations of the Poincare group in 1939, it was known that there are massless and massive representations of integer and half-integer spin [4]. But experimentally there was no need to go beyond spin 1/2 until the end of 1950s when massive higher spin resonances were found in strong interaction physics. ${ }^{2}$ Not until the 1970s were massless fields studied theoretically (except being mentioned in passing) - with one notable exception when the gauge theory of free massless fields was constructed by C. Fronsdal [3] and J. Fang and Fronsdal [6]. The exception was S. Weinberg's S-matrix argument [7] from 1963, showing that massless fields of spin greater than 2 cannot generate long-range forces. And experimentally such fields were not - and still are not - seen. The history of this fascinating subject will have its own chapter in the present book.

Free fields are not so interesting in themselves. The standard recipe for interactions followed in many early references was to try to couple massive higher spin fields to electromagnetism and gravity, or later, to couple conserved matter currents to massless higher spin fields. ${ }^{3}$ It did not work out well, as we will have occasion to study in detail. The investigation of self-interactions came even later with the work of Fang and Fronsdal, who explicitly formulated the research program of finding higher spin interactions as deformations of free field theories [8]. This program - designated as the generalized Gupta program (after S. Gupta, who was one of the pioneers of this approach to gravity) - was a generalization of earlier work to construct gravitational interactions in that manner.

If a starting time for positive results in the study of massless higher spin selfinteractions can be found, it is presumably in the early 1980s with the work of

1 There is actually what can be considered as a co-founder of the subject: E. Majorana with the paper [2] from 1932, pre-dating Dirac.

2 See, for instance, [5], Chapter 21.

3 It can be argued that coupling to gravity is an absolute requirement so that having free higher spin fields would be impossible in principle. 
I. Bengtsson, L. Brink and myself, of F. A. Berends, G. J. H. Burgers and H. van Dam and of E. S. Fradkin and M. Vasiliev. But I think it is safe to say that during most of its history, the theory of higher spin fields, in particular massless fields, has been considered to be very difficult, fraught with consistency problems, experimentally not relevant and perhaps even a totally misguided endeavor. Still a small number of researchers pursued the subject up until the first years into the new millennium when new and young researchers were attracted to it. In the mid 2010s, it was a thriving subject with an established community of researchers working on it. A slight cooling off may be observed as we move into the 2020s. Much has been done during the last 35 years, but the subject is still far from closed. What will happen in the future, we have to wait and see. The basic higher spin problem - researching consistent interactions and investigating their role in nature - is one of those problems that are relatively easy to state, yet so hard to solve, and therefore allures the brave, or foolhardy.

\section{The present state of the subject}

The present state of the subject offers a strange picture. The Vasiliev theory is a background independent formulation of higher spin theory that can be expanded around an anti-de Sitter background (AdS), but apparently not around a Minkowski background. ${ }^{4}$ It partly solves the interaction problem by the so-called "gauging" approach which works very well for spin 1 , can be made to work for spin 2 , but for higher spin forces a number "workarounds" that threatens to remove - in my opinion - the resulting theoretical construction from the basic intuition of the original problem. This is of course a rather common fate to fall upon fundamental questions in theoretical physics, but here it is aggravated by the lack of experimental input. Barring that there may be data, perhaps cosmological, that we do not yet interpret as pertaining to higher spin, there is no phenomenological guidance, except the non-occurrence of higher spin gauge fields at presently attainable energy scales.

For quite a long time, the interaction problem could be considered to be essentially solved in AdS. The problems with expanding the theory around Minkowski space was taken as an indication - sometimes even as a proof - that interacting higher spin gauge theories do not exist in flat space-time. The dominating approach in Minkowski space, the "deformation" approach, also called the Noether procedure or the Fronsdal program, ${ }^{5}$ has been very slow in producing positive results, instead leading to severe difficulties at the quartic level of interaction. It should be noted, though, that the gauging approach does not escape the need for deformation. ${ }^{6}$

\footnotetext{
4 "AdS" is often used as a shorthand for 'Anti-de Sitter space-time'. "dS" is used for "de Sitter". 5 What Fang and Fronsdal called the "generalized Gupta program".

6 Gauging is, in a sense that will be clarified, basically a kinematical procedure of making a global symmetry local. It remains to find the self-interactions of the introduced gauge fields. Here, one is helped by the gauge algebra, but it does not provide the full dynamics.
} 
From another point of view, particle physics is done in flat background, and coming from this environment, it would seem natural to look for higher spin theory in flat space. But to get to higher spin, one must first pass spin 2 - that is gravity - which is naturally interpreted as a curved space-time theory. So it can be argued that flat space higher spin theory is not very natural to consider. On the other hand, gravity can be viewed as a highly nonlinear theory of spin 2 fields, and that theory is not likely to go unaffected by an eventual higher spin theory. Phenomenologically, it seems that we are living in a de Sitter universe, rather than an anti-de Sitter universe. This fact confounds the question even more, if one has hopes for finding a role for higher spin gauge fields in nature. We just do not know enough.

Many readers may have heard that there are quite a few no-go results that rule out either the existence, or at least the relevance, of higher spin theories. Of lately, new difficulties having to do with locality issues has been discovered, both in the Minkowski and the AdS approaches to the theory. These issues are still researched at frontiers of the subject. These problems, and the no-go results, concern the theory of interacting higher spin fields, and belong to a planned second volume of the present work. In the present work, we will mainly treat the free, noninteracting theory, which is of course a prerequisite for any attempt at studying interactions.

\section{A personal note}

On a personal note, in the very late 1980s, after having worked on higher spin theory since my graduate studies, I was discouraged by the difficulties, and this coupled to a lack of new ideas and a wish to spend my time with my children as well as pursuing other intellectual interests, made me temporarily withdraw from the subject. This explains why I did not write a single paper for 14 years. I was not completely idle though, doing calculations for the drawer. ${ }^{7}$ Then in 2003, I saw papers "rediscovering" what I had done in 1986-1988, apparently unaware of my work. Of this, I was irritated, but also glad to see the new interest and I decided to take up the subject again. I was very happy to be able to make a come-back. ${ }^{8}$ When some years later, I started to meet the new researchers in the field, I felt very welcomed back, in particular by Mikhail Vasiliev, who I had actually met only once before, at Ingemar Bengtsson's place in Göteborg some time in the late 1980s.

There were a few exceptions to the relative lack of interest during the 1990s, apart, of course, from M. A. Vasiliev's own work on the AdS formulation of higher spin theory. One is the work of E. Sezgin and P. Sundell [10] starting in 1998. Higher spin excitations also did turn up, and were discussed, in the context of membrane theory and infinite

7 I tried to do BRST theory for singletons in AdS space-time, in that way hoping to find another approach to higher spin fields. This was inspired by the Christian Fronsdal paper [9].

8 This was to some extent helped by the presence of the arXive and TeX-ing, that made "home research" feasible. The engineering school I was working at had no tradition at all in theoretical physics. 
dimensional algebras, but they were not the main subject of focused interest. This was the high tide of superstring theory, and higher spin gauge field theory was definitely at the fringe of theoretical physics. But strangely enough, massless higher spin fields were to turn up in string theory in connection to the AdS/CFT conjectures. That gave a boost to the interest in the Vasiliev theory. ${ }^{9}$

Now, with the subject fairly well established as an interesting part of theoretical physics to pursue, it may be a good time to try and condense and explicate parts of it in book form. The present book came about by a very unlikely - and funny - coincidence. In January 2015, I was at the AMS/MMA Joint Mathematics Meeting in San Antonio, Texas, and gave a presentation on a calculus textbook I was writing with a colleague. When browsing the exhibition hall where the publishers were showing their books, I happened upon the De Gruyter stand. I was leafing through a book when I was approached by Konrad Kieling who was reading my name tag. Now "Bengtsson" is a quite common name in Sweden but not so internationally. Konrad asked be if I knew Ingemar Bengtsson, which I of course did since we were working on higher spins in the 1980s as graduate students and post-docs. This lead to talk about how Konrad knew about Ingemar and about what I was doing and eventually to the question if I had ever thought about writing a book about higher spin. The idea had actually crossed my mind - at least in the form of a review article - but it was very far from being realized. My guarded answer was "yes". After this encounter, the idea started to take on concrete form in the course of an exchange of emails during the spring of 2015. I must take the opportunity to thank my (book writing) colleague and professor in mathematics, Dragu Atanasiu, for prompting me to go to San Antonio.

\section{Audience}

I have tried to make this book accessible to graduate students who have had standard courses in classical mechanics, special and general relativity and quantum mechanics and some quantum field theory. ${ }^{10}$ Although massless higher spin fields and particles are yet to find their proper place in a fundamental description of nature, I have tried nevertheless to connect the subject to basic physical intuition.

When planning the book, I played the game of imagining how some other generic author might write a book on higher spin, and then tried to deviate from that imagined book. In this way, my text would not compete with other eventual texts, but rather be a complement to them. This is my basic philosophy of doing research and writing: I'm not that interested in redoing what others have already done or written. I guess in the end, the book I'm presenting here simply reflects my understanding and point of

9 The subjects mentioned in this paragraph: membrane theory, infinite dimensional algebras, superstring theory, AdS/CFT conjectures and the Vasiliev theory, are all very extensive. I refrain from providing any fair subset of references at this point.

10 Roughly where I was myself when getting started on higher spins in 1983. 
view (and to be honest, lack of knowledge) of this very fascinating subject. However, inevitably much of the material is of course standard for the subject - as is appropriate - but hopefully the approach and emphasis is a bit different. I hope the book contains much of what you need to know to get started on the subject. Apart from technical skills, you will need ideas.

\section{Contents}

The book was initially planned as one volume, but due to the size of the subject, and the time it takes to write about it, the book is divided into two volumes: the free theory in the present Volume 1, and the interacting theory in the planned Volume 2. Higher spin theory has indeed become a very large subject with many aspects and approaches. Even if I had the knowledge and understanding to write about it all, even two volumes would hardly cover it. Rather than trying to write a full review of the whole subject, I have tried to focus on a number of parts of the subject, treating them quite thoroughly. The guiding principle has been to write a text that is concrete and computational, physical and intuitive and conceptual and abstract. The present Volume 1 has the following contents.

Chapter 1 is an introduction. I try to motivate the subject and put it in context, as well as introducing the basic conventions and notation used.

Chapter 2 is historical. I think it is a bit more detailed than has been written on the subject before. I often find historical comments on an area of research both interesting in themselves and helpful for understanding. I hope it will be useful to the reader, too.

Chapter 3 is collection of background knowledge that is essential for working in higher spin theory. Much of the material in this chapter will be used first in Volume 2 on interactions.

Chapter 4 reviews knowledge about the well established theories of spin 1 and spin 2. However, it does so from the perspective of trying to take advantage of such knowledge when approaching the higher spin problem.

In Chapter 5, we come to higher spin theory itself. Here, I treat the free field theory in its various formulations. The focus is on the Minkowski space-time theory.

In Chapter 6, we develop the basics of the light-front approach to massless higher spin fields. Again, as a preparation for a review of what is known about higher spin interactions on the light-front. With this content, the problems of interacting higher spin fields can be discussed in Volume 2.

\section{Referencing}

The number of papers in some way pertaining to higher spin theory may soon run into the thousands, if they have not already done so. I may have seen many of them, but of those I've seen I must admit I have not read them all, and certainly not worked through all the details. 
I've tried to reference original work as fairly as possible. The position of the subject at the outskirts of theoretical physics has, however, had the effect that ideas and methods have been rediscovered over and over again from different perspectives and in different guises - ostensibly unaware of earlier work, ${ }^{11}$ making back-tracking particularly frustrating. Of topics outside higher spin proper, my referencing has been guided by the wish to help the reader to find relevant information in textbooks and reviews, mostly the ones I have found useful myself.

\section{How the book is written}

As many authors have said before: it may be that I wrote the book that I would myself have liked to read when I started out in the subject. Another philosophy that has guided me is something Ingemar Bengtsson said when we were graduate students together: "A good book must have a point of view.". This I agree with. In my opinion, the readable books are often the ones which are based on a few basic unifying ideas that are pursued - not single-mindedly - but pragmatically. This is not an easy ideal to live up to, specially not in a subject like the present one that is far from mature and that has recently undergone rapid development in various directions by many researchers employing different methods, techniques and formalisms. To review all this material, I find out of my reach. There are many different models depending on space-time dimension, back-ground geometry and symmetry groups. This generality - which is of course interesting in itself since it corresponds to a desire to map out the terrain of possible higher spin theories - easily becomes bewildering. The subject tends to look like botany. ${ }^{12}$ It is implicit in my way of looking at the subject, that there are other points of view that one can stress. These you will certainly find in other texts.

To write is therefore as much about what not to include as to what to include. It is only natural if an author wants to express his/her knowledge of the subject (while in the preface perhaps humbly professing to limited knowledge, as I have already done). One often feels that by leaving things out, one does harm to the subject. However, every student of theoretical physics understands that there are more examples of exact solutions to Newton's equation than a particle in a constant force field or in a harmonic potential, but perhaps one does not want to read about them all, at least not just for now.

I have employed another strategy. Almost everything is done in four space-time dimensions and for small symmetry groups and algebras. Thus rather than using my allotted space to listing and classifying various models, I will use the space to try to explain things thoroughly, following a few lines of thought. I will take a walk through the terrain rather than surveying it. I also adhere to the philosophy that formulas do

11 A fault, which I have myself been guilty of: having seen, even read, but forgot.

12 My apologies to the real world botanists. 
not speak for themselves, there must be a story surrounding them. ${ }^{13}$ I have tried to provide such a story for higher spin theory. ${ }^{14}$

Higher spin field theory exploits quite a few tools from mathematics. Although I had from the outset planned a chapter on such things, it eventually grew to one of the longest. I anyway had to work things through to get signs and factors right, and fix notation. For the expert, the contents of this chapter may seem well known or even trivial. I am not quite sure it is so for every nonexpert reader that picks up the book, or for that matter, to newcomers and graduate students. Explaining well-known things in a separate chapter, allows for a shorter and more succinct treatment of the higher spin theory itself. I also found that writing down some of these things was useful in itself, in that hidden assumptions and glossed over details, came into focus. ${ }^{15}$

Another aspect of the large set of mathematical tools used in the subject, is the occurrence of different notational systems, often confusing, sometimes even conflicting. I have tried to streamline notation, at least to harmonize it, but also kept parallel notational systems (the ones that seem most commonly employed). I will explicate this in Section 1.4, in the historical chapter and in Chapter 3.

As an aside, I would actually say that devising formalism is a neglected part of higher spin theory. Having studied computer science for some years, I came to think in terms of objects and processes. In theoretical physics, objects can be fields, processes can be transformations. A symbol for an object needs to carry enough information for it to be able to - in the circumstance - convey, perhaps not all, but its salient properties. This may vary with the context. A process must be symbolized with enough information for the reader to be able to carry through the intended computations reliably. As we all know, this is no easy task. What works well in the personal notebook, may not work so well in print.

An example of a very well devised formalism is Leibniz's formalism for the differential and integral calculus. This is a formalism that computes almost by itself. It

13 In the hilariously funny book on mathematics teaching [11], M. Kline writes: "Many authors seem to believe that symbols express ideas that words cannot. But the symbolism is invented by human beings to express their thoughts. The symbols cannot transcend the thoughts. Hence, the thoughts should first be stated and then the symbolic version might be introduced where symbols are really expeditious. Instead, one finds masses of symbols and little verbal expression of the underlying thought."

14 Regarding the question of the number of dimensions of space-time and size of groups, let me be honest. Limiting myself to $D=4$ and small groups is not just a matter of limiting the scope of the subject. At a deeper level, it is, and has always been, my contention that the world is actually fourdimensional. Fascinating as the subjects of higher dimensions, supersymmetry and large groups undoubtedly are, they have never really spoken to me. My fascination is focused on four dimensions and small groups where I feel there might be depths still not investigated.

15 Let me also risk a quite personal opinion. In Yang-Mills theory, and even gravity, it seems that one can get away with rather sloppy concepts, since one is corrected by - if not reality - by accepted canon. This will not do in higher spin theory. Clear understanding of fundamental concepts of theoretical physics is needed. That is not the same thing as mathematical rigor. 
does so at the price of not completely hiding, but certainly circumventing, the subtleties of analysis. This may bother a mathematician, but is generally no great issue with a theoretical physicist. That is instead what we like about good formalisms. Leibniz's construction of his formalism for the calculus may have been a stroke of genius or inspiration, but certainly not of good luck. As the story goes, Leibniz spent a lot of time on thinking about effective and transparent formalisms in mathematics and philosophy [12]. ${ }^{16}$

A physics example of a very well devised notational system is Dirac's bra and ket notation for quantum mechanics, introduced in 1939 in [13]. A quote from the first paragraph of the paper says it all.

In mathematical theories, the question of notation, while not of primary importance, is yet worthy of careful consideration, since a good notation can be of great value in helping the development of a theory, by making it easy to write down those quantities or combinations of quantities that are important, and difficult or impossible to write down those that are unimportant.

In research articles, and even in review articles, it is only natural to hurry toward what is new, and focus on recent developments. In a book, even if the subject is extensive, one should have the freedom to dwell on the basics of the subject. If not there, where else?

Many books in theoretical physics make a point about developing the subject logically rather than historically. That is a good point, but it is one-sided. Science is a human endeavor, and history is interesting in itself, and it often - or at least sometimes - can shed light on the logic of the subject. Perhaps striking a balance at $25 \%$ history to $75 \%$ logic, is more productive than a balance $5 \%$ to $95 \%$.

For all these reasons, the book is therefore to a large extent, a book in the "rethinking" tradition. And I must admit, as the author, a "relearning” experience. For the reader who finds the treatment unsystematic: think of it as an exploration of unknown territory, rather than as designing a garden.

Some people like exercises and they may be a crucial part of learning a subject. Myself, I don't, always having preferred to choose for myself what to work through and what to trust. The reader of this book will have to do that, too. There are no regular exercises. But there are questions, often of a conceptual rather that technical nature, in certain places in the text. Sometimes tentative answers are given. Sometimes I may not even know - at the time of writing - the answer myself. Other questions may relate to obscure, but interesting, passages in papers. Still others to reproducing results in historical papers. There are cases where I felt a section risked to develop into tedious detail, deflecting from the main goal, so I relegated some material to a question. Questions of these kinds are marked as shown here. I hope readers who enjoy exercises will appreciate these questions. Sometimes the reader has to figure out for herself what the question is.

16 Lars Brink used to say: “The formalism is smarter than we are.”. In higher spin theory, a smart formalism would certainly be of help. 


\section{Acknowledgments}

First of all, I would like to thank my good friends, Gunnar Orrskog and Bobo Ohlsson, who encouraged me to go for early retirement. Upon getting the book writing contract with De Gruyter, I seriously considered that option. Their example made the decision an easy one.

The book is of course a result of years after years of thinking about, and working on higher spin theory. No doubt conference talks I've listened to, conversations - both real and digital - with researchers in the field, and of course paper reading, have contributed to the contents. In particular, I would like to thank Bo Sundborg and Per Sundell for discussions throughout the years and not dismissing my sometimes somewhat orthogonal views on the subject. I have also benefited from discussions with D. Ponomarev and E. Skvortsov when we have met at various conferences during the writing. Thanks also to N. Boulanger and R. Bonezzi during an informal workshop in Mons in January 2018. The same to E. Skvortsov and K. Mkrtchyan for the invitation to the very good Potsdam workshop in December 2018.

During the writing, I have been helped by e-mail conversations with S. Deser, S. Ouvry, M. Tsulaia and G. Barnich. Christian Fronsdal has kindly answered questions and read a draft of the book. So has Lars Brink done, saving me from some embarrassing errors. Ingemar Bengtsson read a draft, found quite a few errors, but otherwise wrote encouraging comments! Remaining errors are of course my responsibility.

Furthermore, I would like to thank the librarians at the University College of Borås for the efficient help with acquiring references, even after having retired from my teaching position at the college. My former co-author and colleague Mats Desaix picked them up and mailed them to me. I found several old books on theoretical physics in the antiquarian bookshop Faust in Göteborg. One of them was the Corson book which turned out to be a gold mine when writing parts of the historical chapter.

The financial support from Stiftelsen Längmanska kulturfonden has been very helpful. 
